\title{
THREE-DIMENSIONAL TORIC MORPHISMS WITH ANTI-NEF CANONICAL DIVISORS
}

\author{
HIROSHI SATO
}

\begin{abstract}
In this paper, we classify projective toric birational morphisms from Gorenstein toric 3-folds onto the 3-dimensional affine space with relatively ample anti-canonical divisors.
\end{abstract}

\section{Contents}

\begin{tabular}{|lr}
\hline 1. Introduction & 1 \\
\hline 2. The definition of local toric Fano varieties & 2 \\
\hline 3. Gorenstein local toric Fano 3-folds & 4 \\
\hline References & 14 \\
\hline
\end{tabular}

\section{INTRODUCTION}

The classification of toric (weak) Fano varieties is an important problem in the toric geometry. It is well-known that the number of Gorenstein toric Fano $d$-folds up to isomorphisms is finite for any $d$ and Gorenstein toric Fano $d$-folds are classified for $d \leq 4$ (see [B1], WW], [B2] and [S] for smooth cases, and see [Ko, [KS1] and [KS2] for Gorenstein cases). In this paper, as a local version of this problem, we consider the following problem which was posed by Watanabe:

Problem 1.1. Classify all the projective toric birational morphisms $f: X \rightarrow \mathbb{A}^{3}$ such that $X$ is smooth and $K_{X}$ is a relatively anti-nef divisor.

By considering this problem up to flops, Problem 1.1 is essentially equivalent to the following problem:

2000 Mathematics Subject Classification. Primary 14M25; Secondary 14E05.

Key words and phrases. Toric varieties, birational morphisms.

The author is partly supported by the Grant-in-Aid for JSPS Fellows, The Ministry of Education, Science, Sports and Culture, Japan. 
Problem 1.2. Classify all the projective toric birational morphisms $f: X \rightarrow \mathbb{A}^{3}$ such that $X$ is Gorenstein and $-K_{X}$ is a relatively ample divisor.

We solve Problem 1.2 under one more assumption that the image of the exceptional set of $f$ is the origin (see Definition 2.1). One of the difficulties of this problem is that the number of such morphisms up to isomorphisms is infinite (see Remark 2.5), while the number of genuine Gorenstein toric Fano 3-folds up to isomorphisms is finite as above. However, we obtain the explicit description of the classification. There are three classes of such morphisms (see Theorem 3.11).

The content of this paper is as follows: In Section 2 we give the definition of local toric Fano varieties. Then, we translate the properties of local toric Fano varieties into the properties of fans. Moreover, we consider the 2-dimensional version of Problem 1.2. Section 3 is devoted to the classification of Gorenstein local toric Fano 3-folds, which is a partial answer to Problem 1.2, The explicit classified list of Gorenstein local toric Fano 3-folds will be given.

Acknowledgments. The author would like to thank Professor Keiichi Watanabe for introducing him to this problem and giving useful comments. He also would like to thank Professors Osamu Fujino and Masataka Tomari for advice and encouragement.

\section{The DEFINITION OF LOCAL TORIC FANO VARIETIES}

First of all, we prepare the notation. For the fundamental properties of the toric geometry, see [F] and $[\mathrm{O}]$.

Let $N:=\mathbb{Z}^{d}$ and $M:=\operatorname{Hom}_{\mathbb{Z}}(N, \mathbb{Z})$ the dual group. The natural pairing $\langle\rangle:, M \times N \rightarrow \mathbb{Z}$ is extended to a bilinear form $\langle$,$\rangle :$ $M_{\mathbb{R}} \times N_{\mathbb{R}} \rightarrow \mathbb{R}$, where $M_{\mathbb{R}}:=M \otimes_{\mathbb{Z}} \mathbb{R}, N_{\mathbb{R}}:=N \otimes_{\mathbb{Z}} \mathbb{R}$. Let $X=X_{\Sigma}$ be a Gorenstein toric $d$-fold associated to a fan $\Sigma$ in $N$. Let $\mathrm{G}(\Sigma)$ be the set of primitive generators of 1-dimensional cones in $\Sigma$, and put $\mathrm{G}(\sigma):=\sigma \cap \mathrm{G}(\Sigma)$ for a cone $\sigma \in \Sigma$. For $\sigma \in \Sigma$, we put $F_{\sigma}:=$ $\operatorname{Conv}(\mathrm{G}(\sigma))$ and $\Gamma_{\sigma}:=\operatorname{Conv}(\mathrm{G}(\sigma) \cup\{0\})$, where $\operatorname{Conv}(T)$ is the convex hull for any subset $T \subset N_{\mathbb{R}}$. For a $d$-dimensional cone $\sigma \in \Sigma$, there exists the unique element $m_{\sigma} \in M$ such that the hyperplane $H_{\sigma} \subset N_{\mathbb{R}}$ generated by $\mathrm{G}(\sigma)$ is defined by $m_{\sigma}$, that is,

$$
H_{\sigma}=\left\{n \in N_{\mathbb{R}} \mid\left\langle m_{\sigma}, n\right\rangle=-1\right\} .
$$

We will use these notation throughout this paper.

Definition 2.1. Let $X=X_{\Sigma}$ be a Gorenstein toric $d$-fold. $X$ is called a local toric Fano (resp. weak Fano) $d$-fold if there exists a projective 
birational toric morphism $f: X \rightarrow \mathbb{A}^{d}$, where $\mathbb{A}^{d}$ is the $d$-dimensional affine space, $-K_{X}$ is $f$-ample (resp. $f$-nef) and the image of the exceptional set of $f$ is the origin.

Remark 2.2. Smooth local toric Fano 3-folds and 4-folds were studied in [S] and [C], respectively. In these papers, the final assumption that the image of the exceptional set of $f$ is the origin was not necessary.

Let $\left\{e_{1}, \ldots, e_{d}\right\}$ be the standard basis for $N, \sigma_{0}:=\operatorname{Cone}\left(e_{1}, \ldots, e_{d}\right)$ and $\Sigma_{0}$ the fan whose cones are all the faces of $\sigma_{0}$. Then, the fan corresponding to $\mathbb{A}^{d}$ is $\Sigma_{0}$. The following is a characterization of local toric Fano $d$-folds using the properties of fans. The proof is similar as the case of genuine toric Fano $d$-folds.

Proposition 2.3. Let $X=X_{\Sigma}$ be a Gorenstein toric d-fold. $X$ is a local toric weak Fano d-fold if and only if $|\Sigma|=\sigma_{0}, \tau \in \Sigma$ for any $\tau \in \Sigma_{0} \backslash\left\{\sigma_{0}\right\}$ and

$$
\Gamma_{\Sigma}:=\bigcup_{\sigma \in \Sigma} \Gamma_{\sigma}
$$

is a convex subset in $N_{\mathbb{R}}$. On the other hand, $X$ is a local toric Fano $d$-fold if and only if $|\Sigma|=\sigma_{0}, \tau \in \Sigma$ for any $\tau \in \Sigma_{0} \backslash\left\{\sigma_{0}\right\}$ and $\Gamma_{\Sigma}$ is a strictly convex subset in $N_{\mathbb{R}}$, that is, $\Gamma_{\Sigma}$ is convex and for any $d$-dimensional cone $\sigma \in \Sigma$ and for any $x \in \Gamma_{\Sigma},\left\langle m_{\sigma}, x\right\rangle=-1$ implies $x \in F_{\sigma}$.

The main purpose of this paper is to give the classification of smooth local toric weak Fano 3-folds up to isomorphisms, which was posed by Watanabe. However, this problem seems very complicated because there may exist many flopping contractions. So, we consider this problem up to flops, that is, we do not classify smooth local toric weak Fano 3 -folds but Gorenstein local toric Fano 3-folds. Then, in principle, we can obtain the classification of smooth local toric weak Fano 3-folds by toric crepant resolutions (see $[\mathrm{OP}$ ).

As the first step for our main result, we consider the classification of Gorenstein local toric Fano surfaces. In this case, there exists a oneto-one corresponding between Gorenstein local toric Fano surfaces and smooth local toric weak Fano surfaces. Namely, we obtain a Gorenstein local toric Fano surface by the anti-canonical morhism of a smooth local toric weak Fano surface, while we obtain a smooth local toric weak Fano surface by the toric crepant resolution of a Gorenstein local toric Fano surface.

Example 2.4 (The classification of smooth local toric weak Fano surfaces). Let $X=X_{\Sigma}$ be a smooth local toric weak Fano surfaces. The 
morphism $f: X \rightarrow \mathbb{A}^{2}$ is factored into a sequence of toric blow-ups:

$$
X=: X_{n} \rightarrow X_{n-1} \rightarrow \cdots \rightarrow X_{1} \rightarrow X_{0}:=\mathbb{A}^{2} .
$$

If $n \neq 0$, then ${ }^{t}(1,1) \in \mathrm{G}(\Sigma)$ because $X_{1} \rightarrow X_{0}$ is a toric blow-up. Similarly, ${ }^{t}(2,1)$ or ${ }^{t}(1,2)$ is contained in $\mathrm{G}(\Sigma)$ if $n \geq 2$. Now, suppose that ${ }^{t}(2,1) \in \mathrm{G}(\Sigma)$. If $\mathrm{G}(\Sigma)$ contains an element ${ }^{t}(s, t) \in N$ such that $s \geq 1$ and $t \geq 2$, then

$$
\operatorname{Conv}\left(0,\left(\begin{array}{l}
1 \\
0
\end{array}\right),\left(\begin{array}{l}
0 \\
1
\end{array}\right),\left(\begin{array}{l}
2 \\
1
\end{array}\right),\left(\begin{array}{l}
s \\
t
\end{array}\right)\right)
$$

contains ${ }^{t}(1,1)$ in its interior. This contradicts the Gorenstein condition. Thus, one can easily check that $X$ is uniquely determined by $n$, that is,

$$
\mathrm{G}(\Sigma)=\left\{\left(\begin{array}{l}
1 \\
0
\end{array}\right),\left(\begin{array}{l}
0 \\
1
\end{array}\right),\left(\begin{array}{l}
1 \\
1
\end{array}\right), \ldots,\left(\begin{array}{l}
n \\
1
\end{array}\right)\right\}
$$

In Figure 1, we describe $\Gamma_{\Sigma}$. For the corresponding Gorenstein local toric Fano surface, we obtain the same convex set.

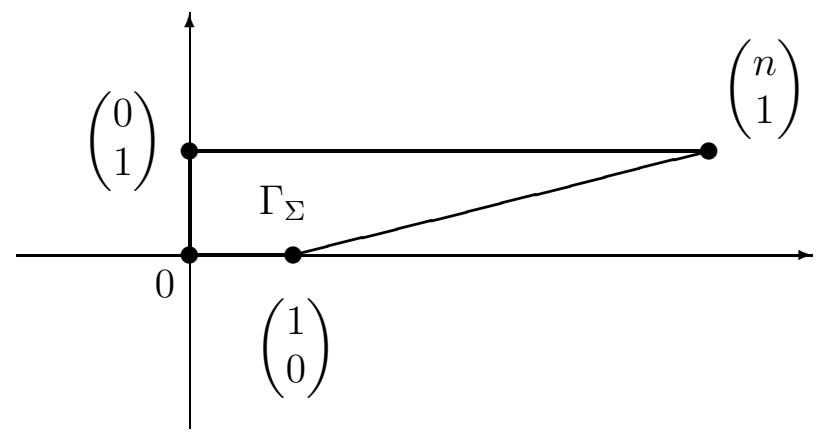

FiguRE 1.

Remark 2.5. As Example 2.4, there exist infinitely many Gorenstein local toric Fano $d$-folds for $d \geq 2$.

\section{Gorenstein LOCAL tORIC FAno 3-FOldS}

In this section, we classify Gorenstein local toric Fano 3-folds.

First of all, we give examples of Gorenstein local toric Fano 3-folds. This large class of Gorenstein local toric Fano 3-folds is important for our classification.

Example 3.1. Let $\left(Z_{1}, Z_{2}, Z_{3}\right)$ be the coordinate for $N_{\mathbb{R}} \cong \mathbb{R}^{3}$. Let $P$ be a convex polytope in the hyperplane $\left(Z_{3}=1\right) \subset N_{\mathbb{R}}$ which satisfies the following conditions: 
TORIC MORPHISMS WITH ANTI-NEF CANONICAL DIVISORS

(1) $e_{3}$ is a vertex of $P$.

(2) For any $x={ }^{t}(a, b, 1) \in P$, we have $a>0$ and $b>0$ unless $x=e_{3}$.

(3) Put the vertices of $P$ be

$$
x_{0}:=e_{3}=\left(\begin{array}{l}
0 \\
0 \\
1
\end{array}\right), x_{1}=\left(\begin{array}{c}
a_{1} \\
b_{1} \\
1
\end{array}\right), \ldots, x_{n}=\left(\begin{array}{c}
a_{n} \\
b_{n} \\
1
\end{array}\right)
$$

clockwise (see Figure 2). We put $x_{n+1}=x_{0}$ for convenience. Then, for any $1 \leq i \leq n+1$, we have the following:

$$
\left\{\begin{array}{lll}
\frac{b_{i}-b_{i-1}}{a_{i}-a_{i-1}} \in \mathbb{Z} & \text { if } & a_{i}-a_{i-1}>0 . \\
\frac{a_{i}-a_{i-1}}{b_{i}-b_{i-1}} \in \mathbb{Z} & \text { if } & a_{i}-a_{i-1} \leq 0 .
\end{array}\right.
$$

For such a polytope $P$, we can define the fan $\Sigma_{P}$ whose maximal cones are

(1) Cone $(P)$,

(2) Cone $\left(e_{2}, x_{i-1}, x_{i}\right)$ for $b_{i}-b_{i-1} \geq-\left(a_{i}-a_{i-1}\right)$,

(3) Cone $\left(e_{1}, e_{2}, x_{i-1}, x_{i}\right)$ for $b_{i}-b_{i-1}=-\left(a_{i}-a_{i-1}\right)$ and

(4) Cone $\left(e_{1}, x_{i-1}, x_{i}\right)$ for $b_{i}-b_{i-1} \leq-\left(a_{i}-a_{i-1}\right)$.

Then, the corresponding toric 3 -fold $X_{P}:=X_{\Sigma_{P}}$ is a Gorenstein local toric Fano 3-fold. To confirm this, we may suffice to say that the maximal cones are Gorenstein. Indeed, the equations of hyperplanes generated by the primitive generators of the above maximal cones are

$$
\text { (1) } Z_{3}=1
$$

$$
\begin{gathered}
\text { (2) } \frac{b_{i}-b_{i-1}}{a_{i}-a_{i-1}} Z_{1}-Z_{2}+\left(-1+b_{i}-\frac{b_{i}-b_{i-1}}{a_{i}-a_{i-1}} a_{i}\right) Z_{3}=-1, \\
(3)-Z_{1}-Z_{2}+\left(-1+a_{i}+b_{i}\right) Z_{3}=-1 \text { and } \\
\text { (4) }-Z_{1}+\frac{a_{i}-a_{i-1}}{b_{i}-b_{i-1}} Z_{2}+\left(-1+a_{i}-\frac{a_{i}-a_{i-1}}{b_{i}-b_{i-1}} b_{i}\right) Z_{3}=-1,
\end{gathered}
$$

respectively. We remark that all the coefficients are integers.

Proposition 3.2. Let $X=X_{\Sigma}$ be a Gorenstein local toric Fano 3fold. $\Sigma$ is associated to a polytope $P \subset\left(Z_{3}=1\right) \subset N_{\mathbb{R}}$ as in Example 3.1 if and only if there exists a 3-dimensional cone $\sigma \in \Sigma$ such that $H_{\sigma}=\left(Z_{3}=1\right)$.

Proof. Suppose that there exists a 3-dimensional cone $\sigma \in \Sigma$ such that $H_{\sigma}=\left(Z_{3}=1\right)$. For any $x \in \mathrm{G}(\Sigma) \backslash \mathrm{G}(\sigma), x$ is contained in $\left(Z_{3}=0\right)$. So, $x$ is either $e_{1}$ or $e_{2}$ by Propsition 2.3. Thus, because of the Gorenstein condition, $X$ is associated to a polytope $P \subset\left(Z_{3}=1\right) \subset N_{\mathbb{R}}$ as in Example 3.1. Another implication is trivial. 


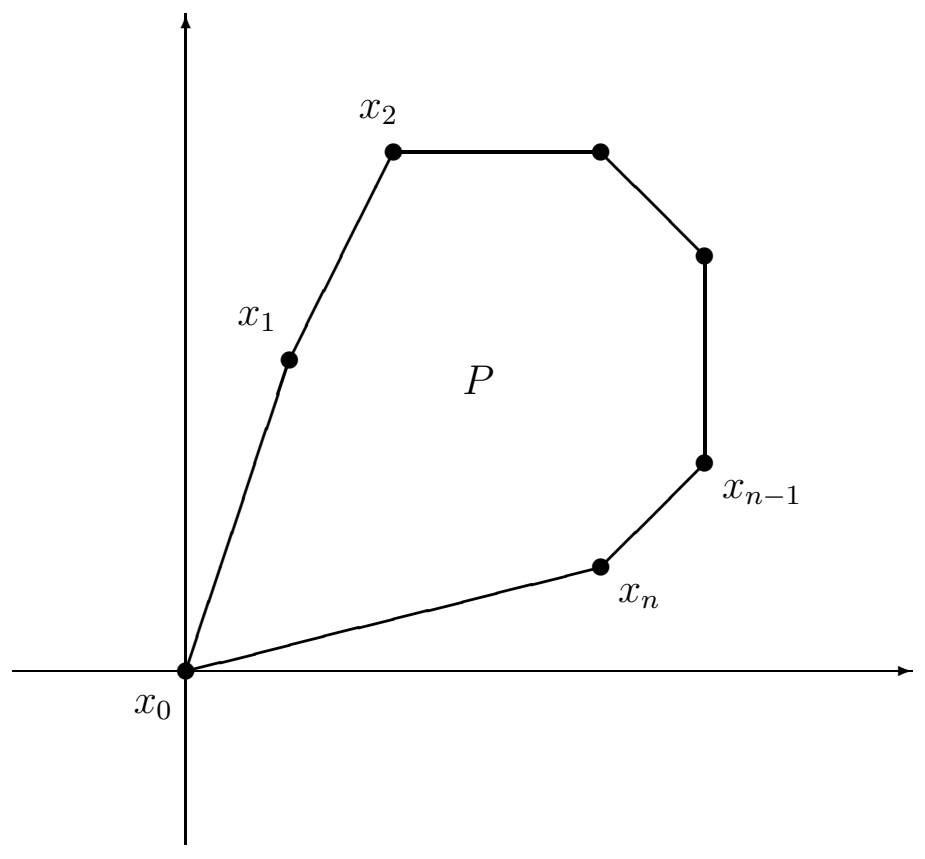

FigURE 2.

Now, we start the classification. The starting point is the following simple lemma.

Lemma 3.3. Let $f: X \rightarrow \mathbb{A}^{d}$ be a projective toric birational morphism from a smooth toric $d$-fold $X=X_{\Sigma}$. If the image of the exceptional set of $f$ is the origin, then either $f$ is an isomorphism or $e_{1}+\cdots+e_{d} \in$ $\mathrm{G}(\Sigma)$.

Proof. $e_{1}+\cdots+e_{d}$ is contained in the relative interior of some cone $\sigma \in \Sigma$. If $\operatorname{dim} \sigma=d$, then $f$ is an isomorphism. So, suppose that $1<\operatorname{dim} \sigma<d$. Then, at least one element $x$ of $\mathrm{G}(\sigma)$ is not contained in $\left\{e_{1}, \ldots, e_{d}\right\}$. Thus, some $(d-1)$-dimensional cone in $\Sigma_{0}$ is not contained in $\Sigma$. This contradicts the fact that the image of the exceptional set of $f$ is the origin. Therefore, $\operatorname{dim} \sigma=1$.

Let $X=X_{\Sigma}$ be a Gorenstein local toric Fano 3 -fold and $\widetilde{X} \rightarrow X$ a toric crepant resolution with $\widetilde{X}=X_{\widetilde{\Sigma}}$. $\widetilde{X}$ is a smooth local toric weak Fano 3-fold. Assume that $f$ is not an isomorphism. Then, by Lemma 3.3 $x_{0}:=e_{1}+e_{2}+e_{3} \in \mathrm{G}(\widetilde{\Sigma})$. Thus, we have the four situations:

(I) $\operatorname{Cone}\left(x_{0}, e_{1}\right) \in \widetilde{\Sigma}$, Cone $\left(x_{0}, e_{2}\right) \in \widetilde{\Sigma}$ and $\operatorname{Cone}\left(x_{0}, e_{3}\right) \in \widetilde{\Sigma}$.

(II) $\operatorname{Cone}\left(x_{0}, e_{1}\right) \notin \widetilde{\Sigma}$, Cone $\left(x_{0}, e_{2}\right) \in \widetilde{\Sigma}$ and $\operatorname{Cone}\left(x_{0}, e_{3}\right) \in \widetilde{\Sigma}$.

(III) Cone $\left(x_{0}, e_{1}\right) \notin \widetilde{\Sigma}$, Cone $\left(x_{0}, e_{2}\right) \notin \widetilde{\Sigma}$ and $\operatorname{Cone}\left(x_{0}, e_{3}\right) \in \widetilde{\Sigma}$. 
(IV) Cone $\left(x_{0}, e_{1}\right) \notin \widetilde{\Sigma}$, Cone $\left(x_{0}, e_{2}\right) \notin \widetilde{\Sigma}$ and $\operatorname{Cone}\left(x_{0}, e_{3}\right) \notin \widetilde{\Sigma}$.

Lemma 3.4. If Cone $\left(x_{0}, e_{i}\right) \notin \widetilde{\Sigma}$, then $x_{i}:=x_{0}+e_{i} \in \mathrm{G}(\widetilde{\Sigma})$.

Proof. We prove the lemma for the case $i=1$.

Suppose that $x_{1} \notin \mathrm{G}(\widetilde{\Sigma})$. Then, we have the equality

$$
x_{1}=\left(\begin{array}{l}
2 \\
1 \\
1
\end{array}\right)=y_{1}+y_{2}
$$

for $\left\{y_{1}, y_{2}\right\} \subset \mathrm{G}(\widetilde{\Sigma})$ which differs from $\left\{x_{0}, e_{1}\right\}$. Therefore, at least one of the 2-dimensional cones in $\Sigma_{0}$ is not contained in $\widetilde{\Sigma}$. This is a contradiction.

In Figure 3. we give the pictures of the above four situations by giving the sections of cones as usual.
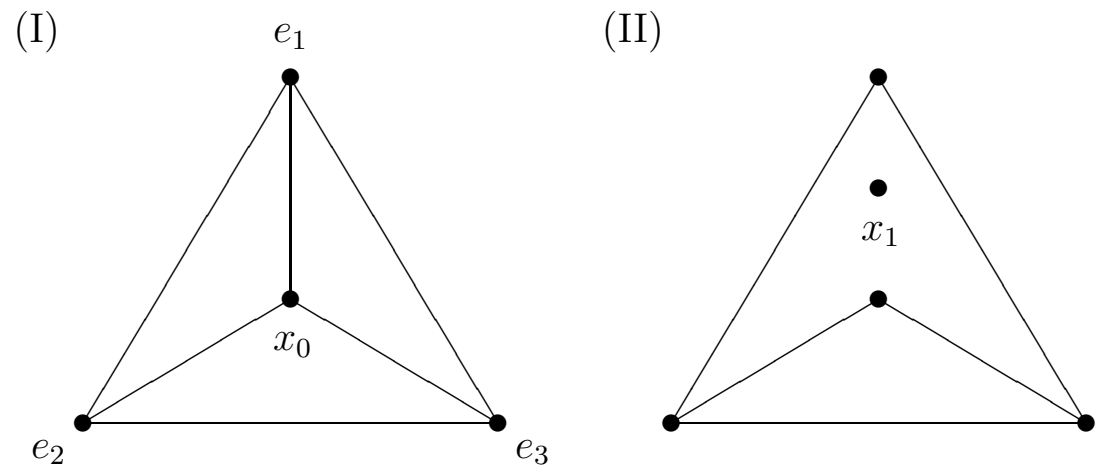

(III)

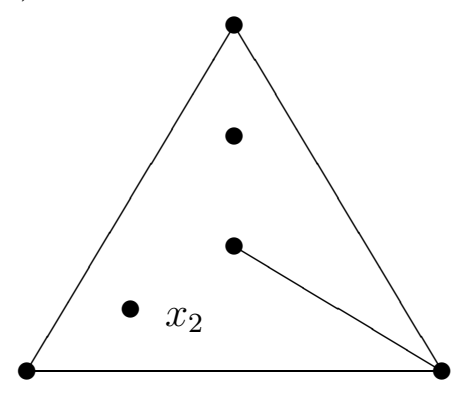

(IV)

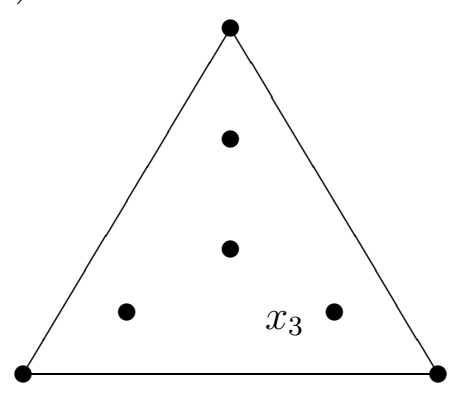

FiguRE 3.

We consider the classification of Gorenstein local toric Fano 3-folds for these four cases. 
(I) In this case, $f$ is factored into

$$
\widetilde{X} \rightarrow X_{1} \rightarrow \mathbb{A}^{3}
$$

where $X_{1} \rightarrow \mathbb{A}^{3}$ is the toric blow-up at the origin. Let $\Sigma_{1}$ be the fan corresponding to $X_{1}$. Then, the following holds.

Lemma 3.5. At least two 3-dimensional cones in $\Sigma_{1}$ are in $\widetilde{\Sigma}$.

Proof. Suppose that Cone $\left(x_{0}, e_{1}, e_{2}\right)$ and Cone $\left(x_{0}, e_{2}, e_{3}\right)$ are not in $\widetilde{\Sigma}$. Then, by Lemma [3.3, $y_{1}:=x_{0}+e_{1}+e_{2}$ and $y_{2}:=x_{0}+e_{2}+e_{3}$ are in $\mathrm{G}(\widetilde{\Sigma})$. However, we have $y_{1}+y_{2}=2 x_{0}+e_{1}+2 e_{2}+e_{3}=3 x_{0}+e_{2}$, and this contradicts the convexity of $\Gamma_{\widetilde{\Sigma}}$.

We may assume that Cone $\left(x_{0}, e_{2}, e_{3}\right)$ and $\operatorname{Cone}\left(x_{0}, e_{3}, e_{1}\right)$ are in $\widetilde{\Sigma}$ by Lemma 3.5. The following proposition completes the classification for the case (I).

\section{Proposition 3.6.}

$$
\Gamma_{\Sigma}=\operatorname{Conv}\left(0, e_{1}, e_{2}, e_{3}, y_{m}:=\left(\begin{array}{c}
m \\
m \\
1
\end{array}\right)\right)
$$

for some integer $m \geq 1$ (see Figure 4).

Proof. If $\widetilde{X} \rightarrow X_{1}$ is not an isomorphism, then there exists $y \in \mathrm{G}(\widetilde{\Sigma})$ which is contained in the interior of $\operatorname{Cone}\left(e_{1}, e_{2}, x_{0}\right)$. So, let

$$
y:=a e_{1}+b e_{2}+c x_{0}=\left(\begin{array}{c}
a+c \\
b+c \\
c
\end{array}\right)
$$

for positive integers $a, b, c$. Suppose that $a>b$. Then, we have

$$
y+b e_{3}=\left(\begin{array}{l}
a+c \\
b+c \\
b+c
\end{array}\right)=(a-b) e_{1}+(b+c) x_{0} .
$$

The inequality $1+b<c+a=(a-b)+(b+c)$ contradicts the convexity of $\Gamma_{\widetilde{\Sigma}}$. Therefore, $a=b$. Similarly, the equality

$$
y+a e_{3}=\left(\begin{array}{l}
a+c \\
a+c \\
a+c
\end{array}\right)=(a+c) x_{0}
$$

implies that $c=1$. Finally, by putting

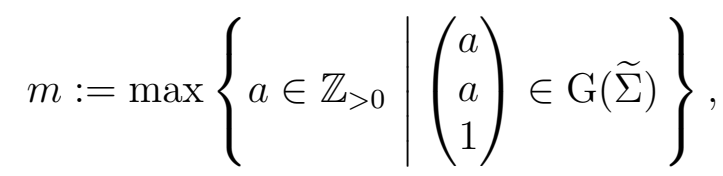


we complete the proof.

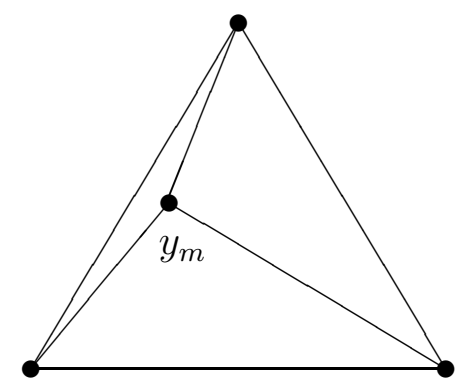

FiguRE 4.

(II) This case is the main part of the classification.

Lemma 3.7. Cone $\left(e_{2}, e_{3}, x_{0}\right) \in \widetilde{\Sigma}$.

Proof. If Cone $\left(e_{2}, e_{3}, x_{0}\right) \notin \widetilde{\Sigma}$, then $e_{2}+e_{3}+x_{0}={ }^{t}(1,2,2) \in \mathrm{G}(\widetilde{\Sigma})$ by Lemma 3.3 .

$$
x_{1}+\left(\begin{array}{l}
1 \\
2 \\
2
\end{array}\right)=\left(\begin{array}{l}
3 \\
3 \\
3
\end{array}\right)=3 x_{0}
$$

implies that $\Gamma_{\Sigma}$ is not convex. Thus, $\operatorname{Cone}\left(e_{2}, e_{3}, x_{0}\right) \in \widetilde{\Sigma}$.

By applying the classification of smooth local toric weak Fano surfaces (see Example 2.4) for the 2-dimensional smooth cone Cone $\left(e_{1}, x_{0}\right)$, we have the following:

Lemma 3.8. One of the following holds:

(IIa) There exists a integer $l \geq 0$ such that

$$
\mathrm{G}(\widetilde{\Sigma}) \cap \operatorname{Cone}\left(e_{1}, x_{0}\right)=\left\{x_{0}, x_{1}, x_{1}+e_{1}, \ldots, x_{1}+l e_{1}, e_{1}\right\} .
$$

$$
\mathrm{G}(\widetilde{\Sigma}) \cap \operatorname{Cone}\left(e_{1}, x_{0}\right)=\left\{x_{0}, x_{0}+x_{1}, x_{1}, e_{1}\right\}
$$

Proof. For case (IIb), there does not exist element in $\mathrm{G}(\widetilde{\Sigma}) \cap \operatorname{Cone}\left(e_{1}, x_{0}\right)$ other than $\left\{x_{0}, x_{0}+x_{1}, x_{1}, e_{1}\right\}$ because the hyperplane spaned by $e_{2}$, $e_{3}$ and $x_{0}+x_{1}$ contains $x_{0}$. 
(IIa) Suppose that there is an element $z \in \mathrm{G}(\widetilde{\Sigma}) \cap \operatorname{Int}\left(\right.$ Cone $\left.\left(e_{1}, e_{2}, x_{0}\right)\right)$, where $\operatorname{Int}(S)$ stands for the interior of $S$. Put $z:=a e_{1}+b e_{2}+c x_{0}$ for positive integers $a, b, c \in \mathbb{Z}_{>0}$.

First, suppose that $c=1$. Then, it is obvious that $\Sigma$ is associated to a polytope $P \subset\left(Z_{3}=1\right)$ which contains $e_{3}, x_{0}, x_{1}+l e_{1}$ and $z$ as in Example 3.1 .

So, suppose that $c \geq 2$. But, this is impossible because $\operatorname{Conv}\left(x_{0}, x_{1}\right)$ is contained in the boundary of $\Gamma_{\Sigma}$. Therefore, there exists no such $z$.

We can apply these arguments for $\left(\operatorname{Cone}\left(e_{1}, e_{3}, x_{0}\right)\right)$ similarly. Thus, in this case, $\Sigma$ is associated to a polytope $P \subset\left(Z_{3}=1\right)$ as in Example 3.1 .

(IIb) First of all, we remark that $\operatorname{Conv}\left(e_{2}, e_{3}, x_{0}+x_{1}\right)$ and $\operatorname{Conv}\left(e_{1}, x_{0}+\right.$ $\left.x_{1}\right)$ are contained in the boundary of $\Gamma_{\Sigma}$. The equations of hyperplanes spaned by $\left\{e_{2}, e_{3}, x_{0}+x_{1}\right\}$ and $\left\{e_{1}, e_{3}, x_{0}+x_{1}\right\}$ are

$$
Z_{1}-2 Z_{2}+Z_{3}=1 \text { and }-Z_{1}+Z_{2}+Z_{3}=1 \text {, }
$$

respectively. Let $\tau$ be the unique 3-dimensional cone in $\Sigma$ such that $e_{1}, e_{2} \in \tau \cap \mathrm{G}(\Sigma)$ and the equation of $H_{\tau}$

$$
Z_{1}+Z_{2}+\alpha Z_{3}=1
$$

where $\alpha \in \mathbb{Z}$. Then, if there is an element $z \in \mathrm{G}(\widetilde{\Sigma}) \cap \operatorname{Int}\left(\operatorname{Cone}\left(e_{1}, e_{2}, x_{0}+x_{1}\right)\right)$, then $z$ is contained in the area

$$
\left\{Z_{1}-2 Z_{2}+Z_{3} \leq 1\right\} \cap\left\{-Z_{1}+Z_{2}+Z_{3} \leq 1\right\} \cap\left\{Z_{1}+Z_{2}+\alpha Z_{3} \leq 1\right\} .
$$

Any point $x$ on the line

$$
L=\left(Z_{1}-2 Z_{2}+Z_{3}=1\right) \cap\left(-Z_{1}+Z_{2}+Z_{3}=1\right)
$$

is described as

$$
x=\left(\begin{array}{c}
3 t-3 \\
2 t-2 \\
t
\end{array}\right)
$$

for $t \in \mathbb{R}$. If $L$ and $H_{\tau}$ intersect at $t_{0} \in \mathbb{R}$, then we have

$$
\alpha=\frac{6}{t_{0}}-5 \text {. }
$$

Obviously, $t_{0} \geq 1$. Thus, we have $1 \leq t_{0} \leq 6$, because $\alpha \in \mathbb{Z}$. Therefore, $z$ is contained in the area

$$
\left\{Z_{1}-2 Z_{2}+Z_{3} \leq 1\right\} \cap\left\{-Z_{1}+Z_{2}+Z_{3} \leq 1\right\} \cap\left\{Z_{1}+Z_{2}-4 Z_{3} \leq 1\right\} .
$$

By considering the area Int (Cone $\left.\left(e_{1}, e_{3}, x_{0}+x_{1}\right)\right)$ similarly, we have the following: 
Proposition 3.9. Put

$$
z_{1}:=\left(\begin{array}{c}
15 \\
10 \\
6
\end{array}\right) \text { and } z_{2}:=\left(\begin{array}{c}
15 \\
6 \\
10
\end{array}\right)
$$

Then, $\Gamma_{\Sigma}$ is contained in

$$
\operatorname{Conv}\left(0, e_{1}, e_{2}, e_{3}, z_{1}\right) \cup \operatorname{Conv}\left(0, e_{1}, e_{2}, e_{3}, z_{2}\right) .
$$

This area is not a convex set (see Figure 5). In particular, the number of Gorenstein local toric Fano 3-folds is finite in this case.

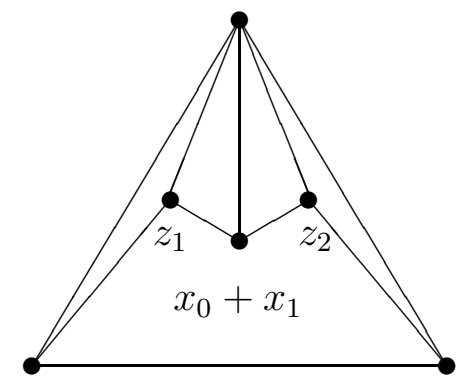

Figure 5.

We can detemine all the possibilities for $\Gamma_{\Sigma}$ as follows: First, we classify lattice polygons in

$$
\operatorname{Conv}\left(e_{2}, e_{3}, z_{1}\right) \cup \operatorname{Conv}\left(e_{2}, e_{3}, z_{2}\right)
$$

which contain $e_{2}, e_{3}$ and $x_{0}+x_{1}$ (there exist exactly 23 such polygons). Then, for any classified polygon $\Delta$, we detemine $\Gamma_{\Sigma}$ such that $F_{\sigma}=\Delta$ for a 3-dimensional cone $\sigma \in \Sigma$. There exist exactly 13 Gorenstein local toric Fano 3-folds in this case. Table 1 is the classified list for these 13 Gorenstein local toric Fano 3-folds. In this table, we give two data: (1) $\mathrm{G}(\Sigma) \backslash\left\{e_{1}, e_{2}, e_{3}\right\}$ and (2) the equation of $H_{\sigma}$ for any 3-dimensional cone $\sigma \in \Sigma$. The following lemma is the key for this calculation.

Lemma 3.10. Let $X=X_{\Sigma}$ be a Gorenstein local toric Fano 3-fold. For any 3-dimensional cone $\sigma \in \Sigma, S_{\sigma}$ contains at least one of $e_{1}, e_{2}$ and $e_{3}$. 
Proof. Let $a Z_{1}+b Z_{2}+c Z_{3}=1$ be the equation of $H_{\sigma}$ for a 3-dimensional cone $\sigma \in \Sigma$. Obviously, $a \leq 1, b \leq 1$ and $c \leq 1$. One can easily confirm that at least one of $a, b$ and $c$ is equal to 1 .

Table 1: Gorenstein local toric Fano 3-folds in the case (IIb)

\begin{tabular}{|c|c|c|c|c|}
\hline & & $(\Sigma) \backslash$ & $\left.e_{1}, e_{2}, e_{3}\right\}$ & $H_{\sigma}^{\prime} \mathrm{s}$ \\
\hline (1) & $\left(\begin{array}{c}15 \\
10 \\
6\end{array}\right)$ & & & $\begin{array}{l}-Z_{1}+Z_{2}+Z_{3}=1, Z_{1}-2 Z_{2}+ \\
Z_{3}=1 \text { and } Z_{1}+Z_{2}-4 Z_{3}=1 .\end{array}$ \\
\hline$(2)$ & $\left(\begin{array}{l}6 \\
4 \\
3\end{array}\right)$ & & & $\begin{array}{l}-Z_{1}+Z_{2}+Z_{3}=1, Z_{1}-2 Z_{2}+ \\
Z_{3}=1 \text { and } Z_{1}+Z_{2}-3 Z_{3}=1\end{array}$ \\
\hline (3) & $\left(\begin{array}{l}3 \\
2 \\
2\end{array}\right)$ & & & $\begin{array}{l}-Z_{1}+Z_{2}+Z_{3}=1, Z_{1}-2 Z_{2}+ \\
Z_{3}=1 \text { and } Z_{1}+Z_{2}-2 Z_{3}=1\end{array}$ \\
\hline$(4)$ & $\left(\begin{array}{l}9 \\
6 \\
4\end{array}\right)$ & and & $\left.\begin{array}{l}5 \\
4 \\
2\end{array}\right)$ & $\begin{array}{l}-Z_{1}+Z_{2}+Z_{3}=1, Z_{1}-2 Z_{2}+ \\
Z_{3}=1, Z_{1}+Z_{2}-4 Z_{3}=1 \text { and } \\
Z_{1}-2 Z_{3}=1\end{array}$ \\
\hline$(5)$ & $\left(\begin{array}{l}5 \\
4 \\
2\end{array}\right)$ & and & $\left.\begin{array}{l}3 \\
2 \\
2\end{array}\right)$ & $\begin{array}{l}-Z_{1}+Z_{2}+Z_{3}=1, Z_{1}-2 Z_{2}+ \\
Z_{3}=1, Z_{1}+Z_{2}-4 Z_{3}=1 \text { and } \\
Z_{1}-Z_{2}=1 .\end{array}$ \\
\hline (6) & $\left(\begin{array}{l}4 \\
3 \\
2\end{array}\right)$ & & $\left.\begin{array}{l}3 \\
2 \\
2\end{array}\right)$ & $\begin{array}{l}-Z_{1}+Z_{2}+Z_{3}=1, Z_{1}-2 Z_{2}+ \\
Z_{3}=1, Z_{1}+Z_{2}-3 Z_{3}=1 \text { and } \\
Z_{1}-Z_{2}=1 .\end{array}$ \\
\hline (7) & $\left(\begin{array}{l}3 \\
2 \\
2\end{array}\right)$ & and & $\left.\begin{array}{l}2 \\
2 \\
1\end{array}\right)$ & $\begin{array}{l}-Z_{1}+Z_{2}+Z_{3}=1, Z_{1}-2 Z_{2}+ \\
Z_{3}=1, Z_{1}+Z_{2}-3 Z_{3}=1 \text { and } \\
Z_{1}-Z_{3}=1 .\end{array}$ \\
\hline (8) & $\left(\begin{array}{l}5 \\
4 \\
2\end{array}\right)$ & and & $\begin{array}{l}2 \\
1 \\
2\end{array}$ & $\begin{array}{l}-Z_{1}+Z_{2}+Z_{3}=1, Z_{1}-3 Z_{2}+ \\
Z_{3}=1, Z_{1}+Z_{2}-4 Z_{3}=1 \text { and } \\
Z_{1}-Z_{2}=1\end{array}$ \\
\hline$(9)$ & $\left(\begin{array}{l}4 \\
3 \\
2\end{array}\right)$ & and & $\left.\begin{array}{l}2 \\
1 \\
2\end{array}\right)$ & $\begin{array}{l}-Z_{1}+Z_{2}+Z_{3}=1, Z_{1}-3 Z_{2}+ \\
Z_{3}=1, Z_{1}+Z_{2}-3 Z_{3}=1 \text { and } \\
Z_{1}-Z_{2}=1\end{array}$ \\
\hline (10) & $\left(\begin{array}{l}6 \\
4 \\
3\end{array}\right)$ & 2 & and $\left(\begin{array}{l}3 \\
2 \\
1\end{array}\right)$ & $\begin{array}{l}-Z_{1}+Z_{2}+Z_{3}=1, Z_{1}-2 Z_{2}+ \\
Z_{3}=1, Z_{1}+Z_{2}-4 Z_{3}=1, \\
Z_{1}-2 Z_{3}=1 \text { and } Z_{2}-Z_{2}=1 .\end{array}$ \\
\hline
\end{tabular}




\begin{tabular}{|l||l}
\hline (11) & $\left(\begin{array}{l}4 \\
3 \\
2\end{array}\right),\left(\begin{array}{l}3 \\
2 \\
2\end{array}\right)$ and $\left(\begin{array}{l}3 \\
2 \\
1\end{array}\right) \cdot \begin{array}{l}-Z_{1}+Z_{2}+Z_{3}=1, Z_{1}-2 Z_{2}+ \\
Z_{3}=1, Z_{1}+Z_{2}-4 Z_{3}=1, \\
Z_{1}-Z_{2}=1 \text { and } Z_{2}-Z_{2}=1 .\end{array}$ \\
\hline$(12)$ & $\left(\begin{array}{l}4 \\
3 \\
2\end{array}\right),\left(\begin{array}{l}3 \\
2 \\
1\end{array}\right)$ and $\left(\begin{array}{l}2 \\
1 \\
2\end{array}\right) \cdot \begin{array}{l}-Z_{1}+Z_{2}+Z_{3}=1, Z_{1}-3 Z_{2}+ \\
Z_{3}=1, Z_{1}+Z_{2}-4 Z_{3}=1, \\
Z_{1}-Z_{2}=1 \text { and } Z_{2}-Z_{2}=1 .\end{array}$ \\
\hline$(13)$ & $\left(\begin{array}{l}3 \\
2 \\
2\end{array}\right),\left(\begin{array}{l}2 \\
1 \\
1\end{array}\right)$ and $\left(\begin{array}{l}2 \\
1 \\
2\end{array}\right) \cdot \begin{array}{l}-Z_{1}+Z_{2}+Z_{3}=1, Z_{1}-3 Z_{2}+ \\
Z_{3}=1, Z_{1}+Z_{2}-3 Z_{3}=1, \\
Z_{1}-Z_{3}=1 \text { and } Z_{1}-Z_{2}=1 .\end{array}$ \\
\hline
\end{tabular}

(III) Since

$$
x_{1}+x_{2}+e_{3}=\left(\begin{array}{l}
3 \\
3 \\
3
\end{array}\right)=3 x_{0},
$$

$\operatorname{Conv}\left(e_{3}, x_{1}, x_{2}\right)$ contains $x_{0}$ in its relative interior. So, $\operatorname{Conv}\left(e_{3}, x_{1}, x_{2}\right)$ is contained in the boundary of $\Gamma_{\Sigma}$. $\operatorname{Conv}\left(e_{3}, x_{1}, x_{2}\right)$ is contained in the hyperplane $\left(Z_{3}=1\right) \subset N_{\mathbb{R}}$. Therefore, by Proposition $3.2, \Sigma$ is associated to a polytope $P \subset\left(Z_{3}=1\right)$ as in Example 3.1

(IV) In this case, $\operatorname{Conv}\left(x_{1}, x_{2}, x_{3}\right)$ is contained in $\Gamma_{\Sigma}$ by Proposition 2.3. So,

$$
x_{1}+x_{2}+x_{3}=\left(\begin{array}{l}
4 \\
4 \\
4
\end{array}\right)=4 x_{0}
$$

implies that $x_{0}$ is contained in the interior of $\Gamma_{\Sigma}$. This is impossible. Therefore, the case (IV) does not occur.

We end this section by summarizing the classification.

Theorem 3.11. Let $X=X_{\Sigma}$ be a Gorenstein local toric Fano 3-fold and $X \neq \mathbb{A}^{3}$. Then, one of the following holds:

(i) For some $m \in \mathbb{Z}_{>0}$,

$$
\Gamma_{\Sigma}=\operatorname{Conv}\left(0, e_{1}, e_{2}, e_{3},\left(\begin{array}{c}
m \\
m \\
1
\end{array}\right)\right)
$$

(see Proposition 3.6]).

(ii) $\Sigma$ is associated to a polytope $P \subset\left(Z_{3}=1\right)$ as in Example 3.1 .

(iii) The other 13 cases (see Table 1). 


\section{REFERENCES}

[B1] V. Batyrev, Toric Fano threefolds, (Russian) Izv. Akad. Nauk SSSR Ser. Mat. 45 (1981), no. 4, 704-717, 927.

[B2] V. Batyrev, On the classification of toric Fano 4-folds, Algebraic geometry, 9. J. Math. Sci. (New York) 94 (1999), no. 1, 1021-1050.

[C] C. Casagrande, Toric Fano varieties and birational morphisms, Int. Math. Res. Not. 2003, no. 27, 1473-1505.

[Fl] W. Fulton, Introduction to toric varieties, Annals of Mathematics Studies, 131, The William H. Roever Lectures in Geometry, Princeton University Press, Princeton, NJ, 1993.

[Ko] R. Koelman, The number of moduli of families of curves on toric surfaces, Thesis, Univ. Nijmegen, 1991.

[KS1] M. Kreuzer and H. Skarke, Classification of reflexive polyhedra in three dimensions, Adv. Theor. Math. Phys. 2 (1998), no. 4, 853-871.

[KS2] M. Kreuzer and H. Skarke, Complete classification of reflexive polyhedra in four dimensions, Adv. Theor. Math. Phys. 4 (2000), no. 6, 1209-1230.

[O] T. Oda, Convex bodies and algebraic geometry, An introduction to the theory of toric varieties, Translated from the Japanese, Ergebnisse der Mathematik und ihrer Grenzgebiete (3) [Results in Mathematics and Related Areas (3)] 15, Springer-Verlag, Berlin, 1988.

[OP] T. Oda and H. S. Park, Linear Gale transforms and Gel'fand-KapranovZelevinsky decompositions, Tohoku Math. J. 43 (1991), 375-399.

[S] H. Sato, Toward the classification of higher-dimensional toric Fano varieties, Tohoku Math. J. 52 (2000), 383-413.

[WW] K. Watanabe and M. Watanabe, The classification of Fano 3-folds with torus embeddings, Tokyo J. Math. 5 (1982), no. 1, 37-48.

Osaka City University Advanced Mathematical Institute, 3-3-138 Sugimoto, Sumiyoshi-Ku, Osaka 558-8585, JaPAN

E-mail address: hirosato@sci.osaka-cu.ac.jp 